\title{
Buprenorphine as a safe alternative to methadone in a patient with acquired long QT syndrome: a case report
}

\author{
I. M. de Jong • G. S. de Ruiter
}

Published online: 29 January 2011

(C) The Author(s) 2011. This article is published with open access at Springerlink.com

\begin{abstract}
A 52-year-old man with a medical history of intravenous drug abuse was admitted to our hospital with syncope due to torsades de pointes (TdP). Two days earlier, he had used methadone. The electrocardiogram showed a prolonged corrected QT interval (QTc) of $600 \mathrm{~ms}$. Continuous telemetry observation showed multiple episodes of TdP. The patient was diagnosed with bradyarrhythmia-induced TdP with acquired long QT syndrome resulting from methadone use. The QTc normalised within 2 weeks after discontinuation of the methadone. In this case of a patient with opioid dependency, there is a reasonable risk of repeated methadone use. Therefore, implantable cardioverter defibrillator or pacemaker implantation is justified but risky because of possible infections when using intravenous drugs. Given the high mortality rates seen in untreated illicit opioid users, this patient needs an alternative pharmacological treatment. Buprenorphine is an opiatereceptor agonist associated with less QTc prolongation. The patient was referred to a rehab clinic and treated with an oral combination of buprenorphine and naloxone (Suboxone). During this therapy, his QTc remained normal.
\end{abstract}

Keywords Long QT · Torsades de pointes .

Buprenorphine $\cdot$ Methadone

I. M. de Jong • G. S. de Ruiter $(\bowtie)$

Onze Lieve Vrouwe Gasthuis,

PO Box 95500, 1090 HM Amsterdam, the Netherlands

e-mail: G.S.deruiter@olvg.nl

\section{Introduction}

Long QT syndrome (LQTS) is a disorder of myocardial repolarisation characterised by a prolonged QT interval on the electrocardiogram (ECG). This syndrome is associated with an increased risk of a characteristic life-threatening cardiac arrhythmia known as torsades de pointes (TdP). The primary symptoms in patients with LQTS include palpitations, syncope, seizures, and sudden cardiac death (SCD). The LQTS may be either genetic or acquired. More than ten different types of congenital LQTS have been identified [1]. Part of the acquired LQTS is also based on LQT-related gene mutations.

\section{Case}

A 52-year-old man with a medical history of intravenous drug abuse and hepatitis $\mathrm{C}$ was admitted to our hospital with TdP. The patient had collapsed and was found on the ground. Two days earlier, he had used methadone, intravenous heroine, and cocaine. The patient had complained of dizziness and syncope before. There was no family history of SCD. Auscultation of the heart did not reveal murmurs. Further physical examination showed no abnormalities besides a haematoma in the right flank. During examination, TdP were observed on the monitor (Fig. 1).

Laboratory results showed no hypokalaemia or hypomagnesaemia. The electrocardiogram showed a prolonged corrected QT interval (QTc) of $600 \mathrm{~ms}$ (Fig. 2). Continuous telemetry observation showed multiple episodes of TdP. Metoprolol $50 \mathrm{mg}$, twice daily, induced mild bradyarrhythmia (50 beats $/ \mathrm{min}$ ) with augmentation of the episodes of $\mathrm{TdP}$. Isoprenaline was started, after which no more TdP were 


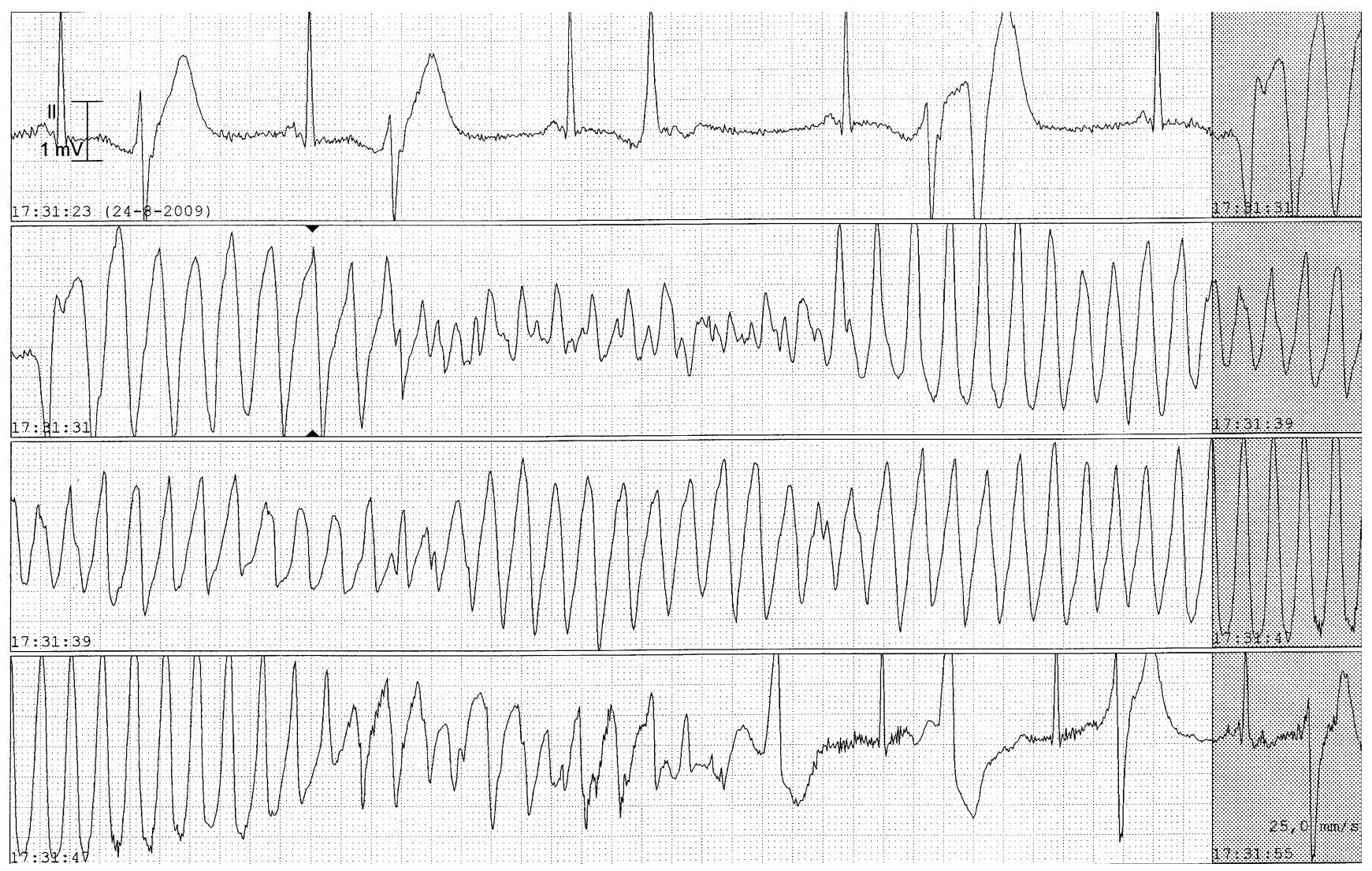

Fig. 1 Torsades de pointes observed on the monitor during examination

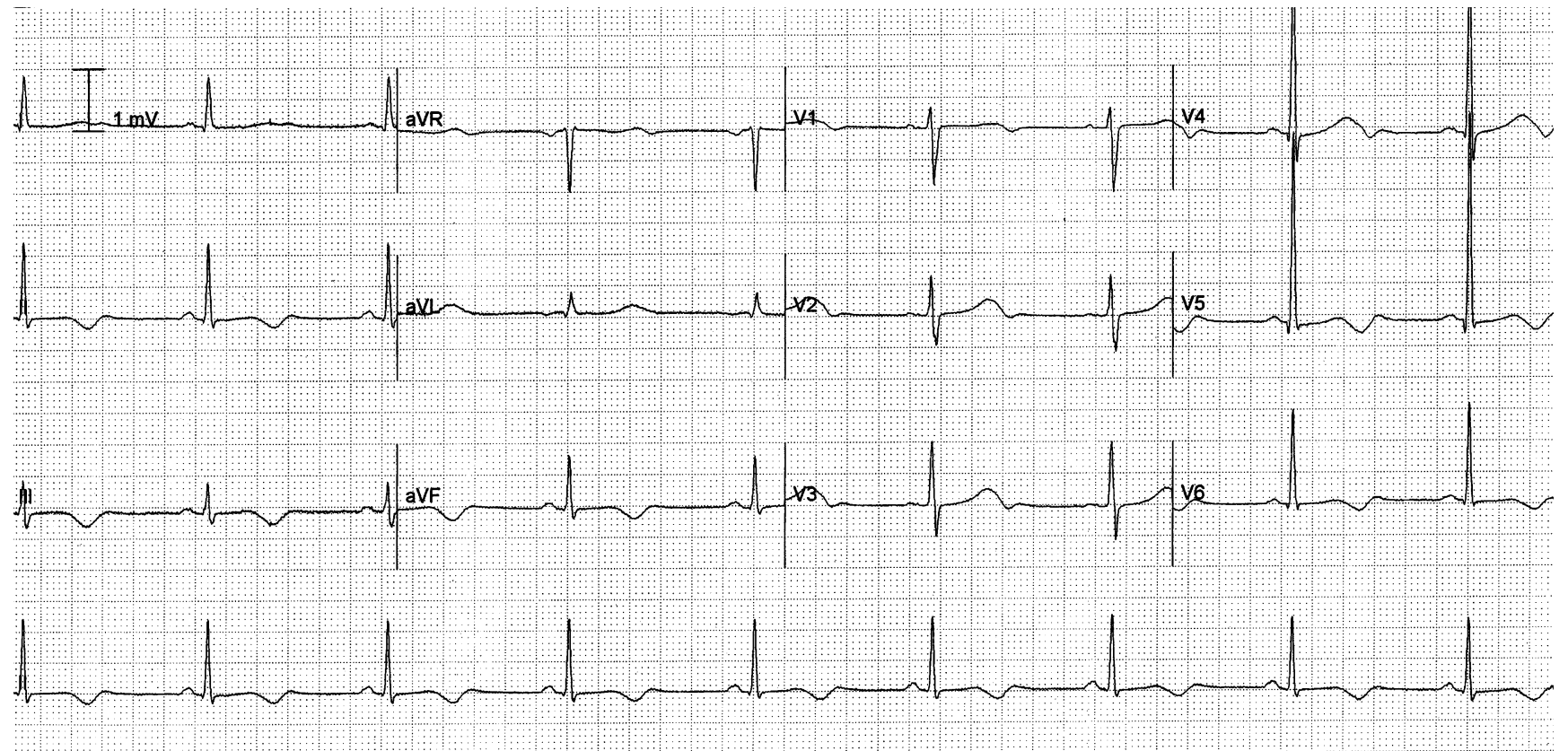

Fig. 2 ECG showing a prolonged corrected QT interval 


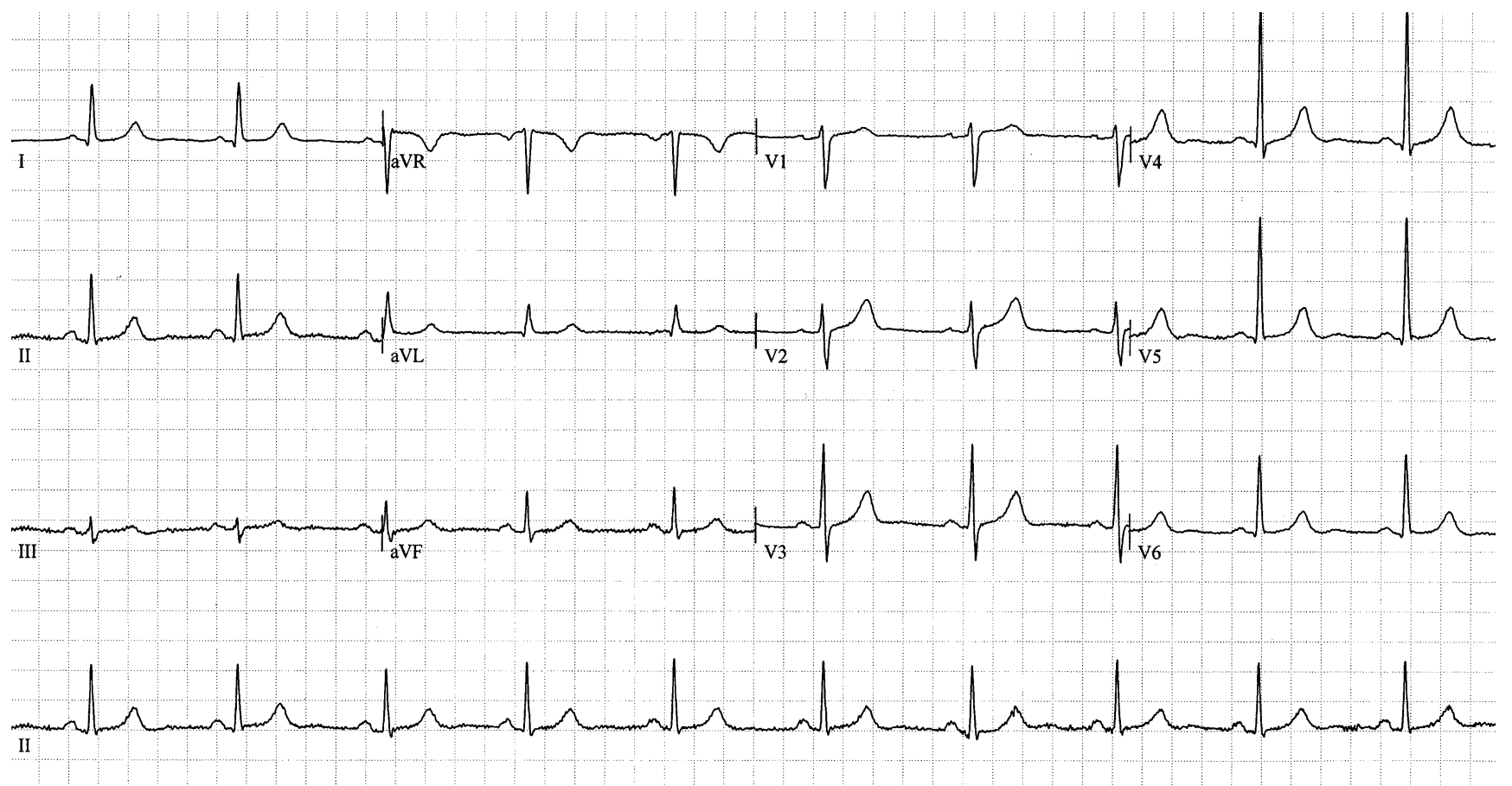

Fig. 3 ECG showing a normalised corrected QT interval

seen. Angiography of the coronary arteries and the left ventricle did not show any abnormalities. The patient was diagnosed with bradyarrhythmia-induced TdP with acquired LQTS resulting from methadone use. The QTc normalised within 2 weeks after discontinuation of the methadone (Fig. 3). The patient was referred to a rehab clinic and treated with an oral combination of buprenorphine and naloxone (Suboxone). During this therapy, his QTc remained normal. Three months later, the patient was still clean. He refused implantable cardioverter defibrillator (ICD) or pacemaker implantation because of possible complications in case of a drug relapse.

\section{Discussion}

Some patients with acquired LQTS appear to have a 'forme fruste' of congenital LQTS, in which a mutation or polymorphism in one of the LQTS genes is clinically silent until the patient is exposed to a particular drug or other predisposing factors. QT prolongation is known to be associated with methadone and is caused by blocking of the human ether-a-go-go-related gene potassium channels mimicking the type 2 of the long QT syndrome [2]. In this case of bradycardia-induced TdP, the initial therapy with $\beta$ blockade was actually contraindicated. The treatment of choice should be intravenous administration of potassium and magnesium, even if blood levels are within the normal range. Magnesium decreases the influx of calcium, thus, lowering the amplitude of early after-deporlarisations [3, 4].
Raising of extracellular potassium concentration increases the efflux of potassium ions from myocardial cells, thus, causing rapid repolarisation [5]. If episodes of $\mathrm{TdP}$ persist, then elevation of the heart rate is indicated, at first instance with isoprenaline, or in case of an insufficient rise of the heart rate, by overdrive pacing. In the long term, overdrive pacing can be performed with a permanent pacemaker or an ICD.

In patients with opioid dependency, there is a reasonable risk of repeated methadone use. Therefore, ICD or pacemaker implantation is justified but risky because of possible infections when using intravenous drugs.

At present, it is not known how often QT prolongation occurs with methadone use, although it appears to be reported infrequently. This may be due to under-recognition, particularly in patients being treated for opioid dependence. Sudden death may be attributed to either narcotic overdose or complications arising from long-term narcotic abuse [6]. Given the high mortality rates seen in untreated illicit opioid users and the clear efficacy of methadone in treating opioid addiction, these patients need an alternative pharmacological treatment. In patients with risk factors for QT interval prolongation, buprenorphine has been used. Buprenorphine is a partial $\mu$-opiate-receptor agonist and a $\mathrm{K}$-opiate-receptor antagonist and is associated with less QTc prolongation.

\section{Conclusion}

Methadone can induce life-threatening TdP in patients with a genetic or acquired LQTS. In patients with opioid 
dependency, there is a reasonable risk of repeated methadone use. Therefore, pacemaker or ICD implantation to avoid bradycardia-induced TdP is justified, but risky because of possible infections when using intravenous drugs. Given the high mortality rates seen in untreated illicit opioid users, these patients need an alternative pharmacological treatment. Buprenorphine can be a safe alternative to methadone in opioiddependent patients with risk factors for QT interval prolongation.

Open Access This article is distributed under the terms of the Creative Commons Attribution Noncommercial License which permits any noncommercial use, distribution, and reproduction in any medium, provided the original author(s) and source are credited.

\section{References}

1. Priori SG, Barhanin J, Hauer RN, et al. Genetic and molecular basis of cardiac arrhythmias: impact on clinical management parts I and II. Circulation. 1999;99(4):518-28.

2. Katchman AN, Mcgroary KA, Kilborn MJ, et al. Influence of opioid agonists on cardiac human ether-a-go-go-related gene $\mathrm{K}$ currents. J Pharmacol Exp Ther. 2002;303:688-94.

3. Tzivoni D, Banai S, Schuger C, et al. Treatment of torsade de pointes with magnesium sulfate. Circulation. 1988;77(2):392-7.

4. Bailie DS, Inoue H, Kaseda S, et al. Magnesium suppression of early afterdepolarizations and ventricular tachyarrhythmias induced by cesium in dogs. Circulation. 1988;77(6):1395-402.

5. Choy AM, Lang C, Chomsky DM, et al. Normalization of acquired QT prolongation in humans by intravenous potassium. Circulation. 1997;96(7):2149-54.

6. Krantz MJ, Lewkowiez L, Hays H, et al. Torsades de pointes associated with very-high-dose methadone. Ann Intern Med. 2002;137(6):501-4. 